\title{
Resistance Noise in Spin Valves
}

\author{
Jørn Foros,${ }^{1}$ Arne Brataas, ${ }^{1}$ Gerrit E. W. Bauer ${ }^{2}$ and Yaroslav Tserkovnyak ${ }^{3}$ \\ ${ }^{1}$ Department of Physics, Norwegian University of Science and Technology, 7491 Trondheim, Norway \\ ${ }^{2}$ Kavli Institute of NanoScience, Delft University of Technology, 2628 CJ Delft, The Netherlands \\ ${ }^{3}$ Lyman Laboratory of Physics, Harvard University, Cambridge, Massachusetts 02138, USA
}

(Dated: May 1, 2019)

\begin{abstract}
Fluctuations of the magnetization in spin valves are shown to cause resistance noise that strongly depends on the magnetic configuration. Due to the applied external field and the dynamic exchange interaction through the normal metal spacer, the electrical noise level of the antiparallel configuration can exceed that of the parallel one by an order of magnitude, in agreement with recent experimental results.
\end{abstract}

PACS numbers: 72.70.+m, 72.25.Mk, 75.75.+a

The dynamics of nanoscale spin valve pillars in which electric currents are flowing perpendicular to the interface planes (CPP) attract much interest [1, 2, 3]. The giant magnetoresistance (GMR) of such pillars of ferromagnetic films separated by normal metals, makes them attractive as future read heads in magnetic hard-disk drives. However, CPP-GMR heads might be flawed by noise. Covington et al. [3] found enhanced low-frequency resistance noise in CPP spin valves. They ascribed this to the spin-transfer torque, i.e., the torque exerted by a spin polarized current on the magnetizations of the ferromagnetic layers [4, [5]. Rebei and Simionato [6] on the other hand, favored micromagnetic disorder as an explanation. More recently, electrical noise measurements have been carried out on CPP nanopillar multilayers with up to 15 magnetic layers [7]. The noise power was found to be reduced by more than an order of magnitude by aligning the magnetizations from antiparallel to parallel in an external magnetic field.

The noise properties of small metallic structures pose challenges for theoretical physics 8] to which ferromagnetism adds a novel dimension [9, 10, 11]. The thermal fluctuations of single domain magnetic clusters have been described already 50 years ago by Brown 12. Recently it has been shown that by contacting the magnet with a conducting environment, the magnetization fluctuations are enhanced compared to the bulk value [11]. Spin valves offer an opportunity to detect the enhanced magnetization noise electrically by the GMR effect, but the new degree of freedom of the detector magnetization complicates the picture in a nontrivial way. The better understanding of the noise properties of CPP nanopillar spin valves reported in the present Letter should therefore be of interest for basic physics as well as applications.

In spin valves, two sources of thermal noise must be taken into account; direct agitation of the magnetizations due to intrinsic processes [12], and thermal spin current fluctuations outside the ferromagnets that affect the magnetizations by means of the spin-transfer torque 11]. Assuming low external bias, spin current shot-noise (due to the discreteness of spin angular momentum) can be disregarded. We show in this Letter that the resulting resistance noise strongly depends on the magnetic configuration. We find that when the ferromagnets are ordered antiparallel, the noise level can indeed be an order of magnitude higher than when they are parallel. Our results thus offer an explanation of the experimental results of Covington et al. [7].

Resistance noise is defined

$$
S\left(t-t^{\prime}\right)=\left\langle\Delta R(t) \Delta R\left(t^{\prime}\right)\right\rangle,
$$

where $\Delta R(t)=R(t)-\langle R\rangle$ is the fluctuation of the resistance at time $t$ from its time-averaged value. We consider a spin valve with two ferromagnetic films with magnetizations $\mathbf{m}_{1}(t)$ and $\mathbf{m}_{2}(t)$ separated by a normal metal. At non-zero temperatures both magnetizations have fluctuating parts $\delta \mathbf{m}_{1}(t)=\mathbf{m}_{1}(t)-\left\langle\mathbf{m}_{1}\right\rangle$ and $\delta \mathbf{m}_{2}(t)=\mathbf{m}_{2}(t)-\left\langle\mathbf{m}_{2}\right\rangle$, due to intrinsic plus spin current noise. The resistance $R(t)$ of the spin valve depends on the angle $\theta$ between the magnetizations. Close to collinear configurations, $R(t)$ can be expanded in the small fluctuations as:

$$
\left.R\left[\mathbf{m}_{1}(t) \cdot \mathbf{m}_{2}(t)\right] \approx R( \pm 1) \mp \frac{1}{2}\left[\delta \mathbf{m}^{\mp}(t)\right]^{2} \frac{\partial R}{\partial \cos \theta}\right|_{P / A P}
$$

where the upper/lower signs hold for the parallel $(\mathrm{P}) /$ antiparallel $(\mathrm{AP})$ orientation, $\delta \mathbf{m}^{\mp}(t)=\delta \mathbf{m}_{1}(t) \mp$ $\delta \mathbf{m}_{2}(t)$, and the differential on the right hand side should be evaluated for $\mathbf{m}_{1} \cdot \mathbf{m}_{2}=\cos \theta=1(\mathrm{P})$ or $\cos \theta=-1$ (AP). Eq. (2) inserted in Eq. (11) expresses the resistance noise in terms of the magnetization fluctuations $\delta \mathbf{m}^{\mp}(t)$. Only the difference between the magnetization vectors $\delta \mathbf{m}^{-}(t)$ induces noise when the magnetizations are parallel, whereas only the sum $\delta \mathbf{m}^{+}(t)$ contributes when antiparallel. The fluctuations $\delta \mathbf{m}^{\mp}(t)$ are the solutions of the stochastic equations of motion for the magnetizations.

To start with, let us consider a single ferromagnetic film isolated from the outside world. The magnetization dynamics in the macro-spin approximation is given by 
the Landau-Lifshitz-Gilbert (LLG) equation:

$$
\frac{d \mathbf{m}}{d t}=-\gamma \mathbf{m} \times\left[\mathbf{H}_{\mathrm{eff}}+\mathbf{h}^{(0)}(t)\right]+\alpha_{0} \mathbf{m} \times \frac{d \mathbf{m}}{d t},
$$

where $\mathbf{m}$ is the unit magnetization vector, $\gamma$ the gyromagnetic ratio, $\mathbf{H}_{\text {eff }}$ the effective magnetic field, $\alpha_{0}$ the Gilbert damping constant, and $\mathbf{h}^{(0)}(t)$ a timedependent random field describing intrinsic thermal agitation. $\mathbf{h}^{(0)}(t)$ has zero average and a white noise correlation function that satisfies the fluctuation-dissipation theorem (FDT) [12],

$$
\left\langle h_{i}^{(0)}(t) h_{j}^{(0)}\left(t^{\prime}\right)\right\rangle=2 k_{B} T \frac{\alpha_{0}}{\gamma M_{s} \mathcal{V}} \delta_{i j} \delta\left(t-t^{\prime}\right) .
$$

Here $i$ and $j$ are Cartesian components, $k_{B} T$ the thermal energy, $M_{s}$ the saturation magnetization, and $\mathcal{V}$ the volume of the ferromagnet. When the ferromagnet is in contact with a conducting environment, two additional related effects may occur. First, when the magnetization precesses, the ferromagnet emits spins into the neighboring conductors ("spin pumping") 13]. In some cases, this can be shown to be equivalent to an enhancement $\alpha^{\prime}$ of the Gilbert damping [13], $\alpha_{0} \rightarrow \alpha_{0}+\alpha^{\prime}$. Secondly, a spinpolarized current (either due to spin pumping or external bias) can exert a torque on the magnetization [4, 5, 14], since its component polarized transverse to the magnetization is absorbed at the interface [15, 16, 17]. This spin-transfer torque may lead to magnetization precession or complete reversal. In spin valves, spin pumping and corresponding spin-transfer torque together couple the LLG-equations of the individual magnets ("dynamic exchange interaction") [18, 19].

Through the spin torque, thermal spin current noise exerts a fluctuating torque on the magnetization vector. As a result, the magnetization noise is increased as compared to the intrinsic noise in an isolated ferromagnet. We have shown that for a single ferromagnet sandwiched by normal metals, the enhancement of the noise is well described by a random field $\mathbf{h}^{\prime}(t)$ similar to, but statistically independent of, the intrinsic random field $\mathbf{h}^{(0)}(t)$ [1]. $\mathbf{h}^{\prime}(t)$ has correlation function equal to Eq. (4) but with the intrinsic damping constant $\alpha_{0}$ replaced by $\alpha^{\prime}$. Thus, the magnetization noise is still governed by the FDT, with the total noise described by the effective random field $\mathbf{h}(t)=\mathbf{h}^{(0)}(t)+\mathbf{h}^{\prime}(t)$ and the total dissipation by the enhanced Gilbert damping constant $\alpha=\alpha_{0}+\alpha^{\prime}$. The appearance of $\alpha^{\prime}$ in this context identifies the thermal spin current noise as the microscopic process that ensures validity of the FDT in the presence of spin pumping [11.

In an unbiased spin valve with a normal metal spacer thicker than the spin-flip diffusion length, the ferromagnets move independently of each other as described by Eq. (3) with $\alpha_{0}, \mathbf{h}^{(0)}$ replaced by $\alpha, \mathbf{h}$. More interesting is a spin valve with a thin spacer through which

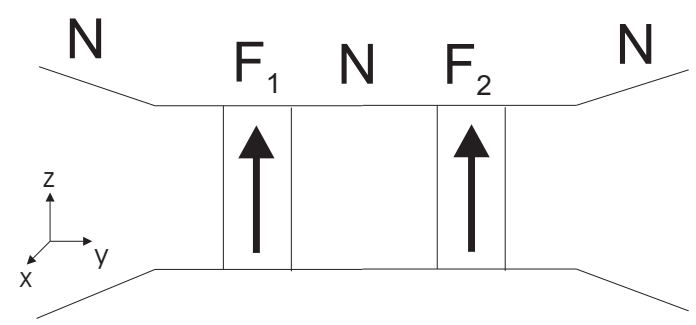

FIG. 1: A spin valve consists of two ferromagnetic thin films $F_{1}$ and $F_{2}$ separated by a normal metal spacer $N$ and connected to normal metal reservoirs. Applied voltages are so small that their effect on the dynamics may be disregarded.

the ferromagnets are allowed to communicate. The dynamic exchange coupling due to spin pumping [18, 19] must then be taken into account, together with the static exchange coupling mediated by electrons in the normal metal spacer, and the static coupling due to the magnetic dipolar interaction. The interlayer static exchange coupling and dipolar coupling can both be described by a Heisenberg coupling $-J \mathbf{m}_{1} \cdot \mathbf{m}_{2}$, where $J$ is the coupling strength, favoring parallel (antiparallel) alignment when $J>0(J<0)$. We consider a spin valve such as the one pictured in Fig. 1 in the regime of significant static and dynamic couplings. Two ferromagnetic thin films are separated by a normal metal spacer and connected to normal metal reservoirs. The ferromagnets are thicker than the magnetic coherence length, so that they perfectly absorb any incoming spin current polarized transverse to the magnetization direction [15, 16, 17]. Spin-flip processes in the middle normal metal are disregarded, which is usually allowed for CPP spin valves. Here we focus on the situation in which the externally applied currents or voltages are sufficiently small to not affect the device dynamics. For simplicity, we take the spin valve to be symmetric (i.e., the two ferromagnets have identical conductance parameters), and only consider parallel and antiparallel magnetic configurations. Assuming that the exchange coupling $J$ is negative, the antiparallel state is the ground state without applied external fields, while the parallel state is achieved by applying a sufficiently strong external magnetic field forcing the magnetizations to align.

Because of the static and dynamic coupling between the ferromagnets, the resistance noise is shown below to vary substantially with the magnetic configuration. The first step is to determine the total noise fields $\mathbf{h}_{k}(t)=$ $\mathbf{h}_{k}^{(0)}(t)+\mathbf{h}_{k}^{\prime}(t)$ for ferromagnet $F_{1}(k=1)$ and ferromagnet $F_{2}(k=2)$. The intrinsic fields $\mathbf{h}_{k}^{(0)}(t)$ are given by Eq. (4), whereas the spin current-induced fields $\mathbf{h}_{k}^{\prime}(t)$ can be calculated using magnetoelectronic circuit theory 15] and the results of Ref. 11]. Requiring conservation of charge and spin in the normal metal spacer [9], and taking into account thermal fluctuations of the distri- 
bution function in the same spacer 9 ], the results are as follows 20]: For both the parallel and antiparallel configurations, the spin current-induced noise fields $\mathbf{h}_{1}^{\prime}(t)$ and $\mathbf{h}_{2}^{\prime}(t)$ are given by

$$
\left\langle h_{k, i}^{\prime}(t) h_{k, j}^{\prime}\left(t^{\prime}\right)\right\rangle=2 k_{B} T \frac{\alpha_{\mathrm{sv}}^{\prime}}{\gamma M_{s} \mathcal{V}} \delta_{i j} \delta\left(t-t^{\prime}\right) .
$$

Here $k=1,2$, and $i$ and $j$ label axes perpendicular to the magnetization direction. Furthermore, $\mathbf{h}_{1}^{\prime}(t)$ and $\mathbf{h}_{2}^{\prime}(t)$ are not statistically independent:

$$
\left\langle h_{1, i}^{\prime}(t) h_{2, i}^{\prime}\left(t^{\prime}\right)\right\rangle=-\left\langle h_{1, i}^{\prime}(t) h_{1, i}^{\prime}\left(t^{\prime}\right)\right\rangle
$$

due to current conservation. As dictated by the FDT, $\alpha_{\mathrm{Sv}}^{\prime}$ is the spin-pumping enhancement of the Gilbert damping in each of the ferromagnets of the spin valve (sv), given by $\alpha_{\mathrm{sv}}^{\prime}=\left(\gamma \hbar \operatorname{Re} g^{\uparrow \downarrow}\right) /\left(8 \pi M_{s} \mathcal{V}\right)\left[19\right.$. Here $g^{\uparrow \downarrow}$ is the dimensionless interface spin-mixing conductance (of which we have disregarded a small imaginary part) 15]. For simplicity, spin pumping and spin current fluctuations into the outer normal metal reservoirs have been disregarded, but can, since the reservoirs are perfect spin sinks, be included simply by increasing the intrinsic Gilbert damping constant $\alpha_{0}$.

We can now calculate the resistance noise, which is given by Eq. (2) inserted in Eq. (1). Assuming that the fluctuations of the magnetization vectors are Gaussian distributed 12], we can employ Wick's theorem 21] and obtain

$$
S_{P / A P}\left(t-t^{\prime}\right)=\frac{1}{2}\left(\frac{\partial R}{\partial(\cos \theta)}\right)_{P / A P}^{2} \sum_{i, j} S_{m_{i}^{\mp} m_{j}^{\mp}}^{2}\left(t-t^{\prime}\right),
$$

for the resistance noise when the magnetizations are parallel (P, upper sign) or antiparallel (AP, lower sign). Here $S_{m_{i}^{-} m_{j}^{-}}\left(t-t^{\prime}\right)=\left\langle\delta m_{i}^{-}(t) \delta m_{j}^{-}\left(t^{\prime}\right)\right\rangle, S_{m_{i}^{+} m_{j}^{+}}\left(t-t^{\prime}\right)=$ $\left\langle\delta m_{i}^{+}(t) \delta m_{j}^{+}\left(t^{\prime}\right)\right\rangle$, and the summation is over all Cartesian components. $S_{m_{i}^{-} m_{j}^{-}}\left(t-t^{\prime}\right)$ and $S_{m_{i}^{+} m_{j}^{+}}\left(t-t^{\prime}\right)$ should be calculated from the LLG equation, Eq. (31), augmented to include the thermal spin current noise, spin pumping, spin torque, and static exchange/dipolar coupling [19]:

$$
\begin{aligned}
\frac{d \mathbf{m}_{k}}{d t}= & -\mathbf{m}_{k} \times\left[\omega_{0} \hat{\mathbf{z}}+\omega_{c}\left(\mathbf{m}_{k} \cdot \hat{\mathbf{x}}\right) \hat{\mathbf{x}}+\omega_{x} \mathbf{m}_{l}+\gamma \mathbf{h}_{k}(t)\right] \\
& +\left(\alpha_{0}+\alpha_{\mathrm{SV}}^{\prime}\right) \mathbf{m}_{k} \times \frac{d \mathbf{m}_{k}}{d t}-\alpha_{\mathrm{SV}}^{\prime} \mathbf{m}_{l} \times \frac{d \mathbf{m}_{l}}{d t}, \quad(8)
\end{aligned}
$$

where $k, l=1,2$ denotes ferromagnets 1 or $2, \omega_{0} \hat{\mathbf{z}}=\gamma \mathbf{H}_{0}$ is an external field applied along the $z$-axis, $\omega_{x}=\gamma J / M_{s} d$ parametrizes the static coupling (where $d$ is the thickness of the ferromagnets), and $\mathbf{h}_{k}(t)=\mathbf{h}_{k}^{(0)}(t)+\mathbf{h}_{k}^{\prime}(t)$ is the total noise field. We have included an in-plane anisotropy field $\omega_{c}\left(\mathbf{m}_{k} \cdot \hat{\mathbf{x}}\right) \hat{\mathbf{x}}=\gamma \mathbf{H}_{c}$ along the $x$-axis. Both ferromagnets are described by the damping parameter $\alpha=\alpha_{0}+\alpha_{\mathrm{sv}}^{\prime}$. The anisotropy field and the negative exchange coupling $\left(\omega_{x}<0\right)$ align the ferromagnets antiparallel along the $x$-axis when the external field is turned off. Then $\mathbf{m}_{k}(t) \approx \pm \hat{\mathbf{x}}+\delta \mathbf{m}_{k}(t)$ for $k=1,2$, where $\delta \mathbf{m}_{k} \approx \delta m_{k, y} \hat{\mathbf{y}}+\delta m_{k, z} \hat{\mathbf{z}}$ are the transverse fluctuations induced by the random noise fields. Linearizing the LLG equation in $\delta \mathbf{m}_{k}$ we can evaluate the magnetization noise $S_{m_{i}^{+} m_{j}^{+}}\left(t-t^{\prime}\right)$ using Eqs. (4), (5) and (6), and find the resistance noise from Eq. (7). When turned on, a large external field enforces a parallel magnetic configuration. Disregarding a sufficiently weak anisotropy field, $\mathbf{m}_{k}(t) \approx \hat{\mathbf{z}}+\delta \mathbf{m}_{k}(t)$ for $k=1,2$, where $\delta \mathbf{m}_{k} \approx \delta m_{k, x} \hat{\mathbf{x}}+\delta m_{k, y} \hat{\mathbf{y}}$. This may be used to find $S_{m_{i}^{-} m_{j}^{-}}\left(t-t^{\prime}\right)$ and subsequently $S_{P}\left(t-t^{\prime}\right)$.

The zero-frequency resistance noise $S_{P / A P}\left(\omega^{\prime}=0\right)=$ $\int d\left(t-t^{\prime}\right)\left\langle\Delta R(t) \Delta R\left(t^{\prime}\right)\right\rangle_{P / A P}$ thus becomes

$$
S_{P / A P}(0)=\frac{2}{\pi}\left(\frac{2 \gamma k_{B} T}{M_{s} \mathcal{V}}\right)^{2}\left(\frac{\partial R}{\partial \cos \theta}\right)_{P / A P}^{2} \int d \omega X_{P / A P}
$$

where

$$
X_{P}=\frac{\left[\omega^{2}+\left(\omega_{t}-\omega_{c}\right)^{2}\right]^{2}+\left(\omega^{2}+\omega_{t}^{2}\right)^{2}+2 \omega^{2}\left(2 \omega_{t}-\omega_{c}\right)^{2}}{2 \alpha_{t}^{-2}\left(\left[\omega^{2}-\omega_{t}\left(\omega_{t}-\omega_{c}\right)\right]^{2}+\omega^{2} \alpha_{t}^{2}\left[2 \omega_{t}-\omega_{c}\right]^{2}\right)^{2}}
$$

for the parallel and

$$
X_{A P}=\left(\frac{\omega^{2} \alpha_{t}+\omega_{c}^{2} \alpha_{0}}{\left[\omega^{2}+\omega_{c}\left(2 \omega_{x}-\omega_{c}\right)\right]^{2}+4 \omega^{2}\left[\omega_{x} \alpha_{0}-\omega_{c} \alpha\right]^{2}}\right)^{2}
$$

for the antiparallel configuration. Due to the fact that the resistance noise goes as square of the magnetization noise (see Eq. (7)), there is an integration over frequency (coming from a Fourier transform) in the above expression. The low-frequency resistance noise is hence not just given by the low-frequency magnetization noise. $\omega_{t}=\omega_{0}+2 \omega_{x}$ and $\alpha_{t}=\alpha_{0}+2 \alpha^{\prime}$ (note the difference with $\alpha=\alpha_{0}+\alpha^{\prime}$ ) correspond to the frequency and damping of the antisymmetric mode $\delta \mathbf{m}^{-}(t)$ in the $\mathrm{P}$ configuration 19]. We have set the external field to zero for the antiparallel configuration, and have assumed the damping to be small, $\alpha^{2} \ll 1$, a condition which is well obeyed by most ferromagnets 19]. The differential $\partial R / \partial \cos \theta$ can be calculated using magnetoelectronic circuit theory [15, 20], and shown to depend weakly on the magnetic configuration [20]. For simplicity, we approximate it in the following to be a constant. The ratio $S_{A P} / S_{P}$ of the noise powers as a function of the strength $-J$ of the static exchange coupling is shown in Fig. 2] for some values of the applied external field in the parallel configuration. Quite reasonably, the noise ratio increases with increasing external field, since this field stabilizes the $\mathrm{P}$ configuration. On the other hand, the noise ratio decreases with increasing coupling strength, because the coupling stabilizes the AP configuration while destabilizing the $\mathrm{P}$ configuration.

Fig. 22 also emphasizes the importance of including spin pumping and spin transfer torque. If disregarded, 


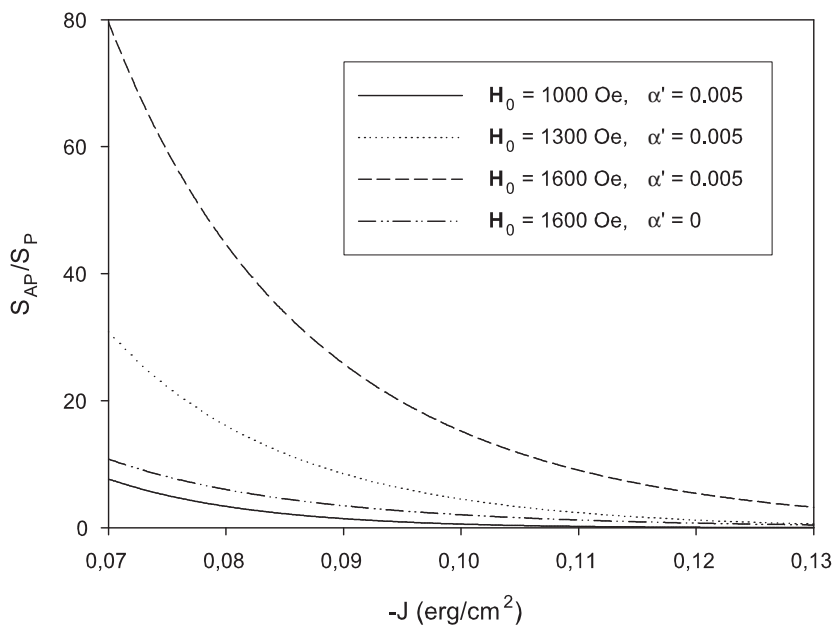

FIG. 2: The ratio $S_{A P} / S_{P}$ of the noise powers as a function of the strength $-J$ of the exchange coupling, for some values of the applied external field in the parallel configuration (in the antiparallel configuration, the external field is zero). The damping has been set to $\alpha_{0}=0.01$ and the anisotropy field to $\omega_{c} / \gamma=10$ Oe.

i.e., $\alpha^{\prime}=0$, the ratio $S_{A P} / S_{P}$ is substantially smaller. To understand this, consider the derivation of, and the expressions for $S_{P}$ and $S_{A P}$ : The noise $S_{P}$ is caused by the antisymmetric mode $\delta \mathbf{m}^{-}(t)=\delta \mathbf{m}_{1}(t)-\delta \mathbf{m}_{2}(t)$. This mode is subject to large fluctuations as well as strongly damped by $\alpha_{t}=\alpha_{0}+2 \alpha^{\prime}[19$, in accordance with the FDT, and as can be seen from Eq. (10). The noise $S_{A P}$ in the AP configuration on the other hand, is caused by the mode $\delta \mathbf{m}^{+}(t)$, which is not as strongly damped. At a first look, it may therefore seem like the FDT should imply larger resistance noise in the $\mathrm{P}$ configuration as compared to the AP. But remember that the resistance noise goes as square of the magnetization noise, resulting in the integration over frequency in Eq. (9). It is this fact, together with the difference in damping of the $\mathrm{P}$ and AP modes (as appearing in the denominators of Eqs. (10) and (11)), that is responsible for the suppression of the resistance noise in the $\mathrm{P}$ configuration as compared to the AP.

We conclude from Fig. 2 that the noise strongly depends on the magnetic configuration, and on the strength of the applied external field in the $\mathrm{P}$ configuration. The noise level can be much higher in the antiparallel configuration than in the parallel, in agreement with the experimental results of Covington et al. 7] on nearly cylindrical multilayer pillars. In these experiments the magnetizations were aligned parallel when the external magnetic field reached about 1500 Oe. It must be noted that whereas we treated spin valves with two ferromagnetic films, Covington et al. dealt with multilayers of $4-15$ magnetic films. It is likely that the difference be- tween the noise properties of bilayers and multilayers is small, as the only local structural difference is the number of neighboring ferromagnets. This assertion is supported by the experiments by Covington et al. that did not reveal strong differences for nanopillars ranging from $4-15$ layers.

We thank Mark Covington for sharing his results prior to publication, and Hans J. Skadsem for discussions. This work was supported in part by the Research Council of Norway, NANOMAT Grants No. 158518/143 and 158547/431, the EU Commission FP6 NMP-3 project 505587-1 "SFINX", and the FOM.

[1] S. I. Kiselev, J. C. Sankey, I. N. Krivorotov, N. C. Emley, R. J. Schoelkopf, R. A. Buhrman, and D. C. Ralph, Nature 425, 380 (2003).

[2] W. H. Rippard, M. R. Pufall, S. Kaka, S. E. Russek, and T. J. Silva, Phys. Rev. Lett. 92, 027201 (2004).

[3] M. Covington, M. AlHajDarwish, Y. Ding, N. J. Gokemeijer, and M. A. Seigler, Phys. Rev. B 69, 184406 (2004).

[4] J. C. Slonczewski, J. Magn. Magn. Mater. 159, L1 (1996).

[5] L. Berger, Phys. Rev. B 54, 9353 (1996).

[6] A. Rebei and M. Simionato, Phys. Rev. B 71, 174415 (2005).

[7] M. Covington, to be published.

[8] Y. M. Blanter and M. Büttiker, Phys. Rep. 336, 1 (2000).

[9] Y. Tserkovnyak and A. Brataas, Phys. Rev. B 64, 214402 (2001).

[10] E. G. Mishchenko, Phys. Rev. B 68, 100409(R) (2003); D. Sánchez, R. López, P. Samuelsson, and M. Büttiker, Phys. Rev. B 68, 214501 (2003); A. Lamacraft, Phys. Rev. B 69, 081301(R) (2004); W. Belzig and M. Zareyan, Phys. Rev. B 69, 140407(R) (2004).

[11] J. Foros, A. Brataas, Y. Tserkovnyak, and G. E. W. Bauer, Phys. Rev. Lett. 95, 016601 (2005).

[12] W. F. Brown, Phys. Rev. 130, 1677 (1963).

[13] Y. Tserkovnyak, A. Brataas, and G. E. W. Bauer, Phys. Rev. Lett. 88, 117601 (2002).

[14] E. B. Myers, D. C. Ralph, J. A. Katine, R. N. Louie, and R. A. Buhrman, Science 285, 867 (1999); J. A. Katine, F. J. Albert, R. A. Buhrman, E. B. Myers, and D. C. Ralph, Phys. Rev. Lett. 84, 3149 (2000).

[15] A. Brataas, Yu. V. Nazarov, and G. E. W. Bauer, Phys. Rev. Lett. 84, 2481 (2000); Eur. Phys. J. B 22, 99 (2001); A. Brataas, G. E. W. Bauer, and P. J. Kelly, Phys. Rep. 427, 157 (2006).

[16] X. Waintal, E. B. Myers, P. W. Brouwer, and D. C. Ralph, Phys. Rev. B 62, 12317 (2000).

[17] M. D. Stiles and A. Zangwill, Phys. Rev. B 66, 014407 (2002).

[18] B. Heinrich, Y. Tserkovnyak, G. Woltersdorf, A. Brataas, R. Urban, and G. E. W. Bauer, Phys. Rev. Lett. 90, 187601 (2003).

[19] Y. Tserkovnyak, A. Brataas, G. E. W. Bauer, and B. I. Halperin, Rev. Mod. Phys. 77, 1375 (2005).

[20] J. Foros, A. Brataas, G. E. W. Bauer, and Y. Tserkovnyak, to be published. 
[21] K. Triantafyllopoulos, The Mathematical Scientist 28, 125 (2003). 